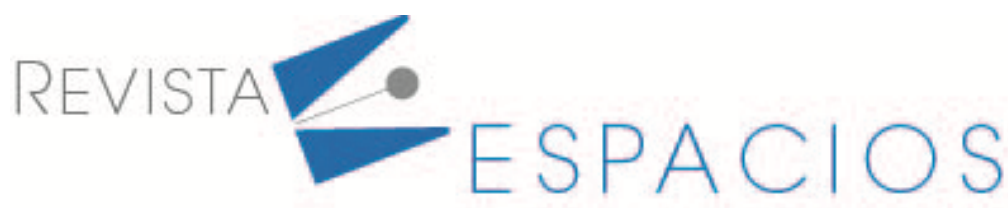

\title{
Calidad de vida laboral del capital humano de la Secretaría de Salud en el sur de Sonora, de un hospital privado
}

\section{Quality of work life of the human capital of the Ministry of Health in southern Sonora, of a private hospital}

\author{
QUIROZ-CAMPAS, Celia Y. ${ }^{1}$ \\ PADILLA-PONCE, Francisco $\mathrm{G}^{2}$ \\ ARMENTA-ZAZUETA, Lizeth. ${ }^{3}$ \\ ACOSTA-MELLADO, Erika I. ${ }^{4}$ \\ MURILLO FÉLIX, Cecilia A. ${ }^{5}$
}

\begin{abstract}
Resumen
El objetivo de la presente investigación fue determinar la calidad de vida laboral del capital humano, percibida por el personal de un hospital privado del Sur de Sonora. La muestra fue de 135 participantes, de los cuales $27.3 \%$ pertenece al área médica, 28.7 a enfermería, 8\% paramédico, 13.3\% administración y $22.7 \%$ puesto afín. Los resultados arrojaron que la calidad de vida incide de manera significativa en antigüedad, área y puesto desempeñado.

Palabras clave: calidad de vida laboral,bienestar individua, hospital y desempeño laboral.
\end{abstract}

\begin{abstract}
The objective of this research was to determine the quality of work life of human capital, perceived by the staff of a private hospital in the South of Sonora. The sample consisted of 135 participants, of which $27.3 \%$ belonged to the medical area, $28.7 \%$ to nursing, $8 \%$ paramedic, $13.3 \%$ administration, and $22.7 \%$ related position. The results showed that quality of life has a significant impact on seniority, area and position held.

Keywords: quality of work life, individual well-being, hospital and work performance.
\end{abstract}

\section{Introducción}

Dentro de los antecedentes de la calidad de vida en el trabajo, se han desarrollado diferentes estrategias a lo largo del tiempo para mejorar el desempeño de los trabajadores, como lo menciona Torres (2017), donde a los trabajadores se les entrega parte de sus ganancias para motivarlos. A pesar de que México pertenece a la Organización para la Cooperación y el Desarrollo Económicos, OCDE, desde 1994 debido a su importancia, las organizaciones no han dedicado el tiempo suficiente a considerar el tema de la calidad de vida laboral de los

\footnotetext{
${ }^{1}$ Dr. en Educación. Profesor Investigador. Instituto Tecnológico de Sonora. celia.quiroz@itson.edu.mx

2 Investigador. Instituto Tecnológico de Sonora. germanpadilla27@icloud.com

${ }^{3}$ Dr. en Educación. Profesor Investigador. Instituto Tecnológico de Sonora. larmenta@itson.edu.mx

${ }^{4}$ Dr. en filosofía con especialidad en administración. Profesor Investigador. Instituto Tecnológico de Sonora. eacosta@itson.edu.mx

${ }^{5}$ Dr. en ciencias económicas administrativa. Profesor Investigador. Instituto Tecnológico de Sonora. cecilia.murillo@itson.edu.mx
} 
trabajadores. Para la Organización Internacional del Trabajo, OIT, elaborar programas que promuevan el trabajo decente de todos es de suma importancia, ya que está fundamentada en el trato decente a los trabajadores. Por otra parte, la Organización Mundial de la Salud, OMS, considera relevante que se evalúe la calidad de vida de los trabajadores y, lo más importante de todo, sensibilizar a los empleadores de que los resultados pueden traer no solo beneficios para el personal, sino también para la productividad de su personal, que es el recurso más importante.

La OCDE es una organización internacional cuya misión es diseñar mejores políticas para el desarrollo de una mejor vida, la cual cuenta con una Iniciativa Better Life, que incluye el bienestar subjetivo, las conexiones sociales, el capital natural y más, que analiza el desempeño de cada país, con la finalidad de conocer la importancia de como es la vida en los difetentes países, manejando la línea de trabajo y calidad del empleo. De manera que el tema de calidad de vida laboral compete a direferentes instituciones de prestigio a nivel nacional e internacional; actualmente es muy común escuchar en diferentes temas de estudio la calidad de vida del capital humano, debido a que este se basa en una percepción que tienen los trabajadores hacia su misma organización sobre la satisfacción o insatisfacción que tienen con respecto al clima laboral en el que se desenvuelven y que viene a evaluar el bienestar y el desarrollo que la empresa les brinda (Cruz, 2018). Para ello es necesario determinar los diferentes factores que influyen en la calidad de vida laboral para determinar si esta afecta o beneficia directamente a los empleados, creando consigo una motivación para mejorar su desempeño en el puesto.

Con este presente estudio se pretende extraer información de primera mano que permita identificar las condiciones y medio ambiente de trabajo en los que se encuentran los empleados, y determinar si cuentan con estructuras que fortalezcan las relaciones entre los miembros que forman parte de la organización, así como el grado de satisfacción que perciben los trabajadores.

La importancia de la presente investigación está en que se aborda el problema del bajo desempeño laboral que con frecuencia se ve en las organizaciones y que muestran las personas en las diferentes áreas, para determinar si cambiando la calidad de vida en el trabajo puede haber un cambio en el desempeño y por tanto resultados totalmente diferentes que permitan a las empresas aumentar su productividad y reducir los tiempos muertos, así como el mejorar el clima laboral.

Además, se pueden generar grandes beneficios para la empresa en estudio, como por ejemplo la mejora de la productividad de los trabajadores, hacer más eficientes los procesos de recursos humanos y enlazar las actividades que tiene el personal con los objetivos y metas de la organización con el fin de optimizar el funcionamiento y asegurar la posición que se tiene dentro del mercado; según Matabanchoy, Álvarez \& Rlobamba (2019), la evaluación de la calidad de vida laboral es muy importante en el desempeño de los profesionales en los hospitales, ya que garantiza el buen cuidado y de la salud en los usuarios; y sobre la base de esto, en futuros trabajos, diseñar un plan estratégico que brinde a la empresa mejorar la calidad de vida del capital humano en el trabajo, el cual, según Patlán (2016), incluye condiciones de trabajo seguro, motivación, bienestar social, capacitaciones enfocadas al crecimiento laboral y desarrollo dentro de las instituciones.

De acuerdo con Patlán la calidad de vida en el trabajo (CVT) comenzó a desarrollarse en el año 1930, pero fue en 1970 cuando su objetivo fue el cumplimiento de la satisfacción de las necesidades que tenían los trabajadores. La razón de ser de la CVT trasciende en la insuficiencia de humanizar las áreas laborales en las que se desarrollan las personas, en crear un entorno democrático mejorando las condiciones de los empleados. Para Hernández et al. (2017) la calidad de vida laboral del capital humano en las áreas de salud es el conjunto de políticas y prácticas que está enlazado con la calidad de los servicios que los trabajadores dan hacia sus clientes, el cual cubre la organización, la satisfacción de los trabajadores y las necesidades que estos tengan en sus respectivos puestos y actividades que desempeñen, y también, muy importante, las expectativas que los empleados tengan hacia organización, derivado de sus expextativas de vida. 
De aquí desprende el concepto de la calidad de vida laboral del capital humano, la cual se puede definir como el proceso cambiante y constante de la organización, para ayudar al desarrollo de los trabajadores dentro de una empresa. Según Quintana et al. (2016), en un estudio llevado a cabo en un hospital privado en Hermosillo, Sonora, menciona que la calidad de vida laboral percibida por los empleados como alta es de tan solo $4.54 \%, y$ el $61.58 \%$ la considera media, y un $32.73 \%$ la percibe como baja; se destaca el bajo porcentaje, el cual al hacer una correlacion se atribuye a que este pequeño grupo de personas son las que están dispuestas a innovar, que tienen un alto sentido de pertenencia hacia la organización, y consideran que tienen apoyo de sus jefes, factores que influyen en la percepción de la calidad de vida laboral.

Un estudio llevado a cabo en los países latinoamericanos en el sector de la salud, menciona que en México el estrés y la sobrecarga laboral se asocian a la insatisfacción laboral, principalmente los horarios y los cambios de turnos, que ocasiona en los empleados altos niveles de agotamiento, el exceso de responsabilidades y el trabajo bajo presión. De la misma manera el estudio fue realizado en Madrid, donde se encontró que el personal de la salud presenta falta de tiempo para su vida personal, consecuencias de salud y estrés. También se encontró que el personal con mayor preparación tiene mejores percepciones de calidad de vida laboral, lo cual refleja una mejor atención a los pacientes y motivación en el trabajo (Caicedo, 2019).

De acuerdo con Münch (2015), el capital humano en todas las organizaciones es fundamental ya que juega un papel muy importante para el logro de las metas y alcance de las ventajas competitivas, de allí que la elección de las personas para laborar en una compañía sea de gran importancia, pues es una de las maneras en que la empresa se desarrolla como se ha planificado.

Por otra parte, también es importante analizar los métodos de evaluación del desempeño, uno de los más destacados es el método $360^{\circ}$, por ser integral al englobar diferentes perspectivas, es decir, desde el jefe directo, compañeros, subordinados, clientes internos y externos, quienes ayudan a realizar la evaluación con el fin de medir el desempeño y evaluar las competencias que tiene cada empleado.

Algunas de las ventajas que tiene este tipo de evaluación es que permite obtener información de cada empleado, y conocer las áreas de mejora; motiva al personal a vivir una cultura organizacional, mejorando la percepción de equidad con procesos transparentes, permitiendo conocer las áreas de oportunidad de cada uno de los colaboradores (Pérez, 2015). Para realizar una evaluación es indispensable dar a conocer a los empleados qué es lo que se va a medir y cuáles son los indicadores que debe de saber para someterse a la evaluación de la performance, con el fin de que conozcan sus responsabilidades y traten de obtener el mejor desempeño posible. Para llevar a cabo esta herramienta es necesario tener bien definido qué indicadores serán los que se evaluarán, quién será el responsable de aplicar el instrumento de evaluación y cómo se va a llevar a cabo, con el único fin de encontrar áreas de oportunidad que le permitan a la empresa mejorar los puntos débiles (Gutiérrez, 2017).

\section{Metodología}

El tipo de investigación es mixta, puesto que dentro del mismo estudio se localizan interrogantes de tipo cualitativo y cuantitativo (Hernández, et al 2014). Este enfoque de métodos mixtos se utiliza empleando como fuente de información una entrevista en la que se incluyen preguntas directas como sexo, edad, puesto, entre otras. Es decir, se recolectan y analizan datos cuantitativos y cualitativos y la interpretación es producto de toda la información en su conjunto.

El primer instrumento empleado para la realización de la investigación fue elaborado por Hernández et al. (2017) considerando como base un estudio de validación de una escala que servirá para la medición de CVL en hospitales privados de Tlaxcala. Tal instrumento se compone de 66 ítems, divididos en dos secciones; dentro de la primera sección se pueden encontrar once apartados que van desde la recolección de datos generales, tales como la 
edad, sexo, estado civil, tipo de contratación, antigüedad en la institución puesto y/o función, ingreso o salario mensual, turno en que labora, área laboral, nivel de estudios y el número de hijos; la segunda parte del instrumento conforman las 55 preguntas.

Dicho instrumento cuenta con tres tipos de validez: contenido, criterio y constructo. La primera se realizó mediante la construcción de un instrumento que mide cuatro dimensiones de la CVL-HP: "bienestar individual", "condiciones y medio ambiente de trabajo", "organización”, y "bienestar logrado a través del trabajo". Una vez elaborado el cuestionario, este se distribuyó a un grupo de psicólogos organizacionales, solicitándoles que validaran con base en los cinco criterios de Moriyama: comprensión de los ítems, aplicabilidad, justificación, definición clara y factibilidad; donde cada criterio se calificó en escala de nada=0 a mucho=3. Las cinco posibles respuestas para cada ítem se diseñaron en escala tipo Likert.

Para el segundo instrumento denominado evaluación del desempeño fue tomado de Zamora (2011) donde utilizó guías de preguntas sobre indicadores de Desempeño y las escalas de Indicadores de Desempeño. Se aplicó en una primera instancia la guía de Preguntas sobre Indicadores de Desempeño del autor Ascary - 2010 CFm.M. Esta guía incluye 30 preguntas enfocadas en 5 variables: Comunicación, Liderazgo, Relaciones Interpersonales, Manejo de Tareas y Trabajo en Equipo; además, cuenta con un sistema de calificación en orden ascendente (NA hasta el número 5 el cual refleja un comportamiento que siempre lo demuestra).

Es importante mencionar que tal guía se presentó al directivo de la empresa a fin de establecer los factores a evaluar y estandarizar los mismos con base a la muestra: considerando las siguientes escalas de Indicadores de Desempeño. 1) de autopercepción, 2) por pares, 3) para uso directivo y 4) para uso de subordinados. Para la escala de Indicadores de Desempeño de Autopercepción, primero se menciona el nombre del puesto que evalúa y cuenta con un sistema de calificación en orden ascendente (NA hasta el número 5 el cual refleja un comportamiento que siempre se demuestra). Aplicable a 6 factores del desempeño (Valores, Comunicación, Liderazgo, Relaciones Interpersonales, Manejo de Tareas, Trabajo en Equipo) los cuales están constituidos con 5 ítems cada uno, en la cual el gerente o encargado de área calificará su propio desempeño en las 6 variables antes mencionadas.

En cuanto a la escala de Indicadores de Desempeño por Pares, cuenta con el nombre del puesto que evalúa e incluye un sistema de calificación en orden ascendente (NA hasta el número 5 el cual refleja un comportamiento que siempre se demuestra), enfocado en las 6 variables anteriormente mencionadas. Está orientado a un sistema de escalonamiento que incluye el nombre del puesto evaluado, en el cual el gerente evaluará el desempeño de sus pares.

En la escala de Indicadores de Desempeño para uso Directivo, incluye un sistema de calificación en orden ascendente (NA hasta el número 5 el cual refleja un comportamiento que siempre se demuestra), en base a las 6 variables ya mencionadas. Se encuentra orientado a un sistema de escalonamiento que incluye el nombre del puesto evaluado, en el cual el directivo evaluará el desempeño de los gerentes y por útimo la escala de Indicadores de Desempeño para uso de subordinados; Incluye un sistema de calificación en orden ascendente (NA hasta el número 5 el cual refleja un comportamiento que siempre se demuestra), con las 30 preguntas referentes a las 6 variables a evaluar. Se hace referencia al nombre del puesto que se va a evaluar, que en este caso es el jefe del subordinado.

Para el desarrollo de esta investigación, se utilizó una metodología donde la ruta de trabajo abarcó las siguientes fases:

1. Se pidió autorización a los accionistas del hospital para poder desarrollar la investigación. 
2. Se seleccionó la muestra, en este caso, la muestra es intencional, ya que se encuestó a todo el personal del hospital, para obtener así la información necesaria para las estadísticas.

3. Se aplicaron los dos instrumentos por tipos de puestos segmentados y se capturó la información del software de análisis de datos SPSS versión 22 con los datos de los encuestados.

4. Se realizó el análisis de los factores que inciden en la calidad de vida laboral y evaluación del desmepeño, en base a pruebas estadísticas para poder tener un resultado de valor.

\section{Resultados}

Los participantes del estudio son 135 sujetos de un hospital privado del Sur de Sonora, donde el género que predominó en los trabajadores que contestaron el instrumento fue el femenino, con el $64.7 \%$, mientras que $25.3 \%$ pertenecía al sexo masculino; $10 \%$ de los participantes que contestaron el instrumento dijo tener menos de 25 años, seguido de los de 25 a 30 años con un $24.7 \%$, de 35 a 45 años el 26.7\%, de 46 a 55 años, 20\%, y 56 o más, $8.7 \%$; lo cual deja en claro que aproximadamente $60 \%$ del personal está considerado relativamente joven. En lo que se refiere al estado civil, $44 \%$ es soltero(a), $23.3 \%$ respondió que es casado(a), seguido de $8.7 \%$ en unión libre, $7.3 \%$ divorciado(a), 5.3\% separado(a) y 1.3\% viudo(a). El personal está distribuido en: $27.3 \%$ al área médica, $28.7 \%$ a enfermería, $8 \%$ paramédico, $13.3 \%$ administración y un $22.7 \%$ puestos afines.

Cuadro 1

Áreas de trabajo

\begin{tabular}{|c|c|}
\hline Área & Porcentaje \\
\hline Médica & $27.3 \%$ \\
\hline Enfermería & $28.7 \%$ \\
\hline Paramédico & $8.0 \%$ \\
\hline Administrativa & $13.3 \%$ \\
\hline Afines & $22.7 \%$ \\
\hline Total & $100 \%$ \\
\hline
\end{tabular}

Fuente: elaboración propia

En cuanto al tipo de contratación, $56.6 \%$ es de base, $6.7 \%$ de confianza, $2.7 \%$ de contrato y $34 \%$ suplente; de los cuales $2.7 \%$ pertenece a directivos; $51.3 \%$ administrativo y el resto a diversas áreas del hospital. Del apartado de la antigüedad de los sujetos en la institución se encontró que $9.3 \%$ tiene menos de un año; $24 \%$ cuenta con entre 1 a 5 años de antigüedad; 34.7\% tiene de 6 a 10 años de antigüedad; $14.7 \%$ de 11 a 15 años; $14 \%$ de 16 a 20 años y solo $3 \%$ cuenta con entre 21 a 25 años de antigüedad en el hospital, y, finalmente, los turnos que laboran, $37.3 \%$ pertenece al matutino; $25.3 \%$, vespertino, $4 \%$ nocturno y $33.4 \%$ mixto.

En cuanto a los resultados obtenidos de la investigación se aplicó el análisis estadístico de fiabilidad de Alfa de Cronbach, con los resultados de la aplicación de instrumento a la empresa en estudio, donde se obtuvo como resultado un .912 .

\section{Resultados del instrumento 1. Calidad de vida laboral}

En el cuadro dos se muestra el resultado del instrumento por dimensiones, donde se puede observar que el mejor evaluado son Condiciones y medio ambiente de trabajo con un 4.56. 
Cuadro 2

Resultados generales del instrumento calidad de vida laboral por dimensión

\begin{tabular}{|c|c|}
\hline Dimensión & Resultado de la media \\
\hline Condiciones y medio ambiente de trabajo & 4.56 \\
\hline Bienestar individual & 4.26 \\
\hline Organización & 4.24 \\
\hline Bienestar logrado a través del trabajo & 4.14 \\
\hline Total general & 4.30 \\
\hline
\end{tabular}

Fuente: elaboración propia

La dimensión mejor evaluada dentro del instrumento 1 fue la organización que se tiene dentro del hospital, donde los participantes destacaron cada una de las diferentes dimensiones. Seguida de esta dimensión está la de bienestar individual, el bienestar logrado a través del trabajo y por último, condiciones y medio ambiente de trabajo. Uno de los aspectos mejor evaluados fue el compromiso que tienen los empleados hacia la organización, lo que contribuye con la imagen que tienen los usuarios del hospital, por lo cual tratan de desarrollar con responsabilidad el rol que les corresponde. Otro aspecto en el que la mayoría de los participantes coincidieron es que el hospital les facilita el equipo y el material necesario para realizar su trabajo, lo cual es un punto muy importante, ya que los empleados se sienten respaldados y les beneficia en el desarrollo de sus actividades.

La variable con el resultado más bajo donde el personal percibe que la institución no reconoce los esfuerzos de eficiencia y preparación con oportunidades laborales, lo cual quiere decir que la empresa no reconoce al empleado y que se dan muy pocos ascensos en los puestos, lo que causa desmotivación entre los participantes. La variable seguida con resultado bajo es que los participantes no se encuentran satisfechos con el área laboral en la que actualmente se encuentran, seguida del turno en el que laboran, percibido como no deseable, y sobre la presión que sienten al realizar su trabajo, que también habla de la inconformidad del trabajador en las diferentes variables de calidad de vida laboral.

En cuanto al cuadro tres sobre el ingreso mensual se observa que el dominante es de 4001 a 8,000, ya que representa $33.3 \%$ del total del resto de los sueldos.

Cuadro 3

Ingreso o salario mensual

\begin{tabular}{|c|c|}
\hline Sueldo & Porcentaje \\
\hline Menos de 4000 & $25.30 \%$ \\
\hline 4001 a 8000 & $33.30 \%$ \\
\hline 8001 a 12000 & $15.40 \%$ \\
\hline 12001 a 16000 & $10 \%$ \\
\hline más de 16001 & $16 \%$ \\
\hline Total & $100 \%$ \\
\hline
\end{tabular}

Fuente: elaboración propia

En cuanto al nivel de estudios se observa que $2 \%$ cuenta con secundaria, $16 \%$ preparatoria, $40 \%$ licenciatura, $31 \%$ especialidad, $6 \%$ maestría y $5 \%$ doctorado, predominando la licenciatura. Ver cuadro 4. 
Cuadro 4

Nivel de estudios

\begin{tabular}{|c|c|}
\hline Escolaridad & Porcentaje \\
\hline Secundaria & $2 \%$ \\
\hline Preparatoria o técnica & $16 \%$ \\
\hline Licenciatura & $40 \%$ \\
\hline Especialidad & $31 \%$ \\
\hline Maestría & $6 \%$ \\
\hline Doctorado & $5 \%$ \\
\hline Total & $100 \%$ \\
\hline
\end{tabular}

Fuente: elaboración propia

En el cuadro 5 se muestra la prueba de normalidad del puesto, área laboral y antigüedad en la institución en cuanto al nivel de estudios del personal del hospital investigado, donde el análisis del nivel de significación se interpreta, si es menor que 0.05 la distribución no es normal, si es mayor que 0.05 la distribución es normal.

Cuadro 5

Pruebas de normalidad

\begin{tabular}{|c|c|c|c|c|c|c|c|}
\hline & \multirow[b]{2}{*}{ Nivel de estudios } & \multicolumn{3}{|c|}{ Kolmogorov-Smirnov ${ }^{\mathrm{a}}$} & \multicolumn{3}{|c|}{ Shapiro-Wilk } \\
\hline & & Estadístico & $\mathrm{gl}$ & Sig. & Estadístico & gl & Sig. \\
\hline \multirow[t]{6}{*}{ Puesto y/o función } & Secundaria &. & 3 &. & . & 3 & . \\
\hline & Preparatoria o técnica & .379 & 24 & .000 & .629 & 24 & .000 \\
\hline & Licenciatura & .368 & 60 & .000 & .643 & 60 & .000 \\
\hline & Especialidad & .417 & 31 & .000 & .661 & 31 & .000 \\
\hline & Maestría & .400 & 10 & .000 & .658 & 10 & .000 \\
\hline & Doctorado & .345 & 7 & .012 & .732 & 7 & .008 \\
\hline \multirow[t]{6}{*}{ Área laboral } & Secundaria &. & 3 &. &. & 3 &. \\
\hline & Preparatoria o técnica & .339 & 24 & .000 & .790 & 24 & .000 \\
\hline & Licenciatura & .230 & 60 & .000 & .842 & 60 & .000 \\
\hline & Especialidad & .252 & 31 & .000 & .794 & 31 & .000 \\
\hline & Maestría & .472 & 10 & .000 & .532 & 10 & .000 \\
\hline & Doctorado & .331 & 7 & .020 & .773 & 7 & .022 \\
\hline \multirow{6}{*}{$\begin{array}{l}\text { Antigüedad en la } \\
\text { institución }\end{array}$} & Secundaria & .385 & 3 &. & .750 & 3 & .000 \\
\hline & Preparatoria o técnica & .264 & 24 & .000 & .854 & 24 & .003 \\
\hline & Licenciatura & .174 & 60 & .000 & .915 & 60 & .000 \\
\hline & Especialidad & .192 & 31 & .005 & .905 & 31 & .010 \\
\hline & Maestría & .189 & 10 & $.200^{*}$ & .926 & 10 & .410 \\
\hline & Doctorado & \begin{tabular}{|l|}
332 \\
\end{tabular} & 7 & .019 & .869 & 7 & .183 \\
\hline \multicolumn{8}{|c|}{ *. Esto es un límite inferior de la significación verdadera. } \\
\hline \multicolumn{8}{|c|}{ a. Corrección de significación de Lilliefors } \\
\hline
\end{tabular}

Fuente: elaboración propia

En el cuadro 6 se puede apreciar la percepción que ven los diferentes sujetos en estudio, donde la percepción más baja se puede ver que está en la del director hacia sus directivos, donde obtuvo el resultado más bajo con $81.47 \%$, y claramente se puede ver que la más alta fue la de subordinados con $92.82 \%$, del cual se puede deducir que es un resultado aceptable, al igual que el de evaluación por pares con un total de $89.02 \%$, seguido de la autopercepción por parte de los directivos con $86.90 \%$. 


\section{Resultados del instrumento 2. Percepción del desempeño de los trabajadores}

Cuadro 6

Resultados Generales de la evaluación del desempeño por puestos

\begin{tabular}{|c|c|}
\hline Rol & Resultado \\
\hline Subordinados & $92.82 \%$ \\
\hline Pares & $89.02 \%$ \\
\hline Autopercepción & $86.90 \%$ \\
\hline Directivo & $81.47 \%$ \\
\hline
\end{tabular}

Fuente: elaboración propia

Estos resultados nos indican que existe una gran diferencia entre la percepción de las diferentes partes, desde los subordinados y del directivo, por lo que vale la pena destacar que la óptica es totalmente diferente, ya que las expectativas entre el director y los subordinados son acordes con lo que les corresponde hacer.

\section{Resultados del auto percepción del desempeño de los trabajadores}

Como se puede observar en el cuadro 7, los resultados de los indicadores de la autopercepción del desempeño la variable mejor percibida es la comunicación, con $90 \%$, cuyo resultado se considera aceptable, seguido de este indicador están el de valores, el cual tiene $89.4 \%$ y se puede percibir que existen los valores como respeto, principios éticos y sobre todo, que se trabaja en confianza, lo cual es considerado un aspecto muy importante para los demás trabajadores, ya que en cualquier empresa los valores pasan a formar parte de ella.

El trabajo en equipo fue la variable que obtuvo $86.2 \%$, la cual está en un área de oportunidad para mejorar, así como la de relaciones intrapersonales, con $85.6 \%$. La variable con un porcentaje menor fue liderazgo, que se percibe entre los trabajadores como la más baja, y es un punto muy importante a mejorar para que se pueda desarrollar dentro de la empresa, ya que esta es una de las habilidades gerenciales que debe de tener el nivel directivo, porque representa una influencia jerárquica en los empleados.

Cuadro 7

Resultado de la escala de Indicadores de Desempeño de

Autopercepción de trabajadores de manera general

\begin{tabular}{|c|c|}
\hline Variable & Porcentaje \\
\hline Comunicación & $90.0 \%$ \\
\hline Valores & $89.4 \%$ \\
\hline Manejo de tareas & $88.6 \%$ \\
\hline Trabajo en equipo & $86.2 \%$ \\
\hline Relaciones interpersonales & $85.6 \%$ \\
\hline Liderazgo & $83.0 \%$ \\
\hline
\end{tabular}

Fuente: elaboración propia

Las variables mejor evaluadas del instrumento 2, fue la comunicación, donde los participantes respondieron que se les escucha cuidadosamente cuando solicitan el apoyo, seguido de que demuestran interés y respeto hacia las otras personas, lo cual habla muy bien de la institución, ya que una de las principales problemáticas que se dan en las organizaciones es la mala comunicación, y en este caso es la mejor evaluada entre los trabajadores. Está seguida de los valores, donde se respondió que en la empresa existen los principios éticos, un punto favorable ya que en la actualidad representa un tema muy importante que beneficia a las empresas y a su imagen. 
La variable seguida es la de manejo de tareas, la cual se encuentra en un punto medio. Por lo cual se afirma que la diversidad de resultados en otros estados de la república, ampara el desarrollo de este tipo de investigación, como la realizada por González et al (2019) sobre calidad de vida laboral en trabajadores de la Salud en Guadalajara, México, donde se encontró más satisfacción por parte del personal, ya que este tipo de estudios tiene una gran importancia para la productividad y eficiencia empresarial.

Las variables con el resultado más bajo son las relaciones interpersonales y el liderazgo, dos aspectos muy importantes en los que tendrá que trabajar la empresa para mejorar estas áreas de oportunidad, esto debido a que los participantes coincidieron en que en la empresa no se crean las condiciones por parte de los directivos que faciliten a cada uno dar lo mejor de sí y donde se evidencia la falta de liderazgo en áreas claves para el óptimo funcionamiento de la empresa.

\section{Resultados de las variables por tipo de puestos}

El cuadro 8 muestra los resultados de las variables vistos desde las diferentes perspectivas; el que obtuvo mejor porcentaje fue la variable de los valores, el cual refleja que existe similitud en la percepción de los diferentes sujetos del estudio, y que además es un buen indicio de que existen valores dentro de la empresa. Se puede ver que los resultados son similares, es decir, no cambian mucho los puntos porcentuales, lo cual quiere decir que los resultados se encuentran en la media, pero que existe el área de mejora. Los factores con los resultados más bajos son el trabajo en equipo y las relaciones interpersonales, lo cual incita a la empresa a tomar medidas que ayuden a los trabajadores a desarrollar buenas relaciones con sus compañeros, y a llevar a cabo estrategias que propicien el trabajo en equipo y la empatía, las cuales son pieza fundamental para un mejor desempeño de los trabajadores y una mejor calidad de vida laboral.

Cuadro 8

Resultados de las variables por tipos de puestos

\begin{tabular}{|c|c|c|c|}
\hline Variable & Directivo & Pares & Subordinados \\
\hline Valores & $88.8 \%$ & $92 \%$ & $93 \%$ \\
\hline Manejo de tareas & $80.0 \%$ & $90 \%$ & $94 \%$ \\
\hline Liderazgo & $79.5 \%$ & $88 \%$ & $93 \%$ \\
\hline Comunicación & $78.7 \%$ & $90 \%$ & $94 \%$ \\
\hline Trabajo en equipo & $78.4 \%$ & $89 \%$ & $84 \%$ \\
\hline Relaciones interpersonales & $76.0 \%$ & $86 \%$ & $91 \%$ \\
\hline
\end{tabular}

Fuente: elaboración propia

\section{Resultados de pares por departamentos}

En el cuadro 9 se puede observar la evaluación por pares, donde cada área evaluó las demás, y la media entre las diferentes áreas fue de $89.02 \%$, resultado considerado aceptable; pero es importante destacar que algunos estuvieron por debajo de la media, como por ejemplo intendencia, relaciones públicas, seguros, facturación, servicios generales, laboratorios y cobranza, siendo intendencia la que obtuvo el resultado más bajo con $72.81 \%$, punto muy importante que deberán ver más en detalle los directivos para mejorar este indicador. El área con mejor desempeño fue contaduría, con 99.67\%, el cual refleja que es el área con mejor desempeño en base a la percepción, seguido de sistemas y banco de sangre (98.72\% y $97.29 \%$ respectivamente). Esta última evaluación es muy importante ya que cada una de las áreas dio su propia perspectiva evaluando a las demás, y en base a esta se puede deducir que existen áreas que se ocupan de mejorar y que trabajan una retroalimentación para poder mejorar la percepción de su desempeño. 
Cuadro 9

Resultados de pares por departamentos

\begin{tabular}{|c|c|}
\hline Departamento & Porcentaje \\
\hline Compras & $89.71 \%$ \\
\hline Enfermería & $92.76 \%$ \\
\hline Banco de sangre & $97.29 \%$ \\
\hline Intendencia & $72.81 \%$ \\
\hline Servicios generales & $83.38 \%$ \\
\hline Laboratorio & $88.36 \%$ \\
\hline Restaurante & $89.95 \%$ \\
\hline Recursos Humanos & $94.26 \%$ \\
\hline Cobranza & $88.81 \%$ \\
\hline Seguros & $81.38 \%$ \\
\hline Contador & $99.67 \%$ \\
\hline Pagos & $96.85 \%$ \\
\hline Encargado de sistemas & $98.52 \%$ \\
\hline Relaciones Públicas & $80.00 \%$ \\
\hline Facturación & $81.61 \%$ \\
\hline Total de Medias & $89.02 \%$ \\
\hline
\end{tabular}

Fuente: elaboración propia

De las 4 diferentes perspectivas (autopercepción, director, subordinados y pares) con las que se abordó la evaluación $360^{\circ}$, la mejor evaluada fue por los subordinados, mismos que evaluaron a sus jefes inmediatos, y la poco mejor fue la del director hacia los encargados de las diferentes áreas, donde se puede notar la perspectiva que tienen los diferentes participantes, pues el director pide lo mejor de cada quien para lograr que se cumplan los objetivos y metas, y el subordinado evaluando desde el área que él observa. Es importante destacar que de los resultados obtenidos en el instrumento de pares el área que obtuvo el resultado más bajo fue la de intendencia, seguida de relaciones públicas, seguros y facturación, mientras que las áreas mejor evaluadas fueron la de contabilidad, sistemas, banco de sangre y pagos.

Para establecer correlaciones entre las variables del instrumento, se aplicó el coeficiente de correlación $r$ de Pearson y se pudo observar una correlación significativa entre la edad de los integrantes de la muestra y el estado civil (véase cuadro 10).

Cuadro 10

Matriz de correlaciones $r$ de Pearson en la muestra total

\begin{tabular}{|c|c|c|c|c|c|c|}
\hline & & Edad & Sexo & Estado civil & Tipo de contratación & Área laboral \\
\hline \multirow[t]{2}{*}{ Edad } & Correlación de Pearson & 1 & .087 & $.211^{*}$ & $-.192^{*}$ & .118 \\
\hline & Sig. (bilateral) & & .376 & .031 & .050 & .231 \\
\hline \multirow[t]{2}{*}{ Sexo } & Correlación de Pearson & .087 & 1 & .001 & -.081 & -.060 \\
\hline & Sig. (bilateral) & .376 & & .996 & .413 & .546 \\
\hline \multirow[t]{2}{*}{ Estado civil } & Correlación de Pearson & $.211^{*}$ & .001 & 1 & .021 & $.203^{*}$ \\
\hline & Sig. (bilateral) & .031 & .996 & & .835 & .038 \\
\hline \multirow{2}{*}{$\begin{array}{c}\text { Tipo de } \\
\text { contratación }\end{array}$} & Correlación de Pearson & $-.192^{*}$ & -.081 & .021 & 1 & .054 \\
\hline & Sig. (bilateral) & .050 & .413 & .835 & & .581 \\
\hline \multirow[t]{2}{*}{ Área laboral } & Correlación de Pearson & .118 & -.060 & $.203^{*}$ & .054 & 1 \\
\hline & Sig. (bilateral) & .231 & .546 & .038 & .581 & \\
\hline
\end{tabular}

Fuente: elaboración propia 


\section{Conclusiones}

El resultado de esta investigación muestra en general el sentir de los diferentes participantes; se puede determinar los puntos en los que el hospital tendrá que enfocar sus estrategias para mejorar, desde la calidad de vida laboral, hasta el desempeño de los trabajadores; es de gran importancia que la empresa conozca sus fortalezas, de las cuales tiene unas que cualquiera pudiera desear tener, pero que tendrá que trabajar para que esas se sigan manteniendo y tratar de convertir las debilidades en fortalezas que beneficien tanto a la organización como a los trabajadores.

\section{Recomendaciones}

El llevar a cabo una investigación de este tipo donde se evalúen aspectos de gran impacto para la organización y contribuya al logro de la razón de ser de compañía; por tal motivo y en base a los resultados obtenidos y a las deducciones generadas a través de los instrumentos se plantean las siguientes recomendaciones:

De acuerdo con los resultados obtenidos, el hospital necesita crear un programa para impulsar al personal con mejor desempeño de la empresa, esto en base a su trabajo, y crear nuevas oportunidades laborales que permitan a los empleados sentirse reconocidos, esto ya sea por reconocimientos, cambio de puestos, bonos y compensaciones, lo cual traería una alta motivación, la cual se vería reflejada en un mejor rendimiento de los trabajadores.

Además, se sugiere al departamento de recursos humanos crear estrategias que permitan que los trabajadores no se sientan presionados ni estresados por las actividades diarias que tienen que realizar, lo cual crearía un mejor ambiente laboral e impactaría significativamente en el desempeño de los trabajadores. Otra recomendación que se le puede brindar a la empresa es realizar constantemente el tipo de evaluación $360^{\circ}$, no solamente en el personal directivo sino tratar de llevar a cabo a todo el personal, esto con el fin de calificar a cada uno de ellos, y clasificar entre altos, medios y bajos, a los altos reconocerlos y con los medios y bajos tratar de capacitarlos en los aspectos más bajos y darle un seguimiento para tratar de optimizar su desempeño. Según Casallas (2016), a la evaluación $360^{\circ}$ busca ayudar a las empresas a explotar al máximo el potencial de cada uno de los trabajadores, de allí la importancia de seguir llevando a cabo este tipo de evaluación dentro de la empresa con el fin de trabajar con las debilidades que se tienen en cada uno de los departamentos.

También es necesario crear una evaluación de cada uno de los trabajadores con el fin de recopilar información sobre el perfil de cada uno, para determinar el área laboral a la que se asignará, ya que muchos de los encuestados comentaron que les gustaría cambiar de departamento o que se sentirían mejor realizando otras actividades, por lo que se le sugiere al departamento de recursos humanos llevar a cabo antes de la contratación, y durante con el fin de determinar si el trabajador se siente conforme y menos estresado en las tareas que le corresponden. De acuerdo a Durán (2010) cuando el entorno laboral es seguro y cómodo los trabajadores se sienten más satisfechos e impacta favorablemente en la calidad de vida laboral.

Con respecto al personal directivo, es necesario capacitarlos sobre el liderazgo y crear estrategias con el fin de desarrollarles la habilidad, ya que esto ayuda al trato con los trabajadores; por ser el comunicador directo de la empresa puede ayudar a que los empleados se sientan más en confianza y respaldados por su jefe inmediato, con trato decente y justo, lo que hará que los trabajadores se sientan comprometidos con la empresa por sentirse bien en su área de trabajo.

Realizando una comparación entre el estudio aplicado por Medina \& Olivares (2018) se llega a la misma conclusión y recomendación, es decir, se sugiere a la empresa en estudio seguir con la implementación de evaluación de los instrumentos de evaluación de desempeño y calidad de vida laboral, con el fin de capacitar a 
los empleados en los indicadores con menor puntaje, además de trabajar en el diseño de estrategias que permita tener bien motivado al personal, ofreciendo incentivos y reconocimientos al personal con mejor desempeño y como empresa tratar de llevar un seguimiento de la percepción que los empleados tienen sobre la calidad de vida en el área laboral.

Otro de los autores que coinciden en los resultados del presente estudio es Baquerizo (2015), quien a su vez comparte que el analizar las diferentes percepciones que tienen los empleados con los distintos puestos que van desde directores, jefes, colaboradores, permite a la empresa mejorar los procesos que se llevan a cabo, y ver las debilidades que tiene, con el fin de trabajar en ello y convertirlas en fortalezas, que venga a retroalimentar al personal y optimizar resultados de cada una de las áreas.

Por tal se puede concluir que a partir de este estudio se pudieran hacer nuevas investigaciones en otro tipo de empresas, lo cual ayudaría a conocer de primera mano la percepción que los trabajadores tienen hacia su trabajo, y, lo más importante, sobre la base de resultados y datos recopilados, llevar a cabo una toma de decisiones acertada.

\section{Referencias bibliográficas}

Baquerizo, L. (Diciembre de 2015). Evaluación de 360 grados del desempeño laboral en los trabajadores del área de operaciones de la Clínica Good Hope, Miraflores, 2015. Recuperado de https://repositorio.upeu.edu.pe/bitstream/handle/UPEU/575/Lucero_Tesis_bachiller_2015.pdf?sequence $=1 \&$ isAllowed=y.

Caicedo Fandiño, H. Y. (2019). Calidad de vida laboral en trabajadores de la salud en países latinoamericanos: meta-análisis. Signos. Investigación en Sistemas de Gestión, 11(2), 41-62. Recuperado de Doi: https://doi.org/10.15332/24631140.5081

Casallas, D. (2016). Aplicación de la evaluación $360^{\circ}$ como herramienta para alcanzar el éxito profesional del recurso humano en las empresas. Recuperado de https://repository.unimilitar.edu.co/handle/10654/14298

Cruz, J. (2018). La calidad de vida laboral y el estudio del recurso humano: una reflexión sobre su relación con las variables organizacionales. Redalyc. Recuperado de https://www.redalyc.org/jatsRepo/646/64659525004/html/index.html

Durán, M. (2010). Bienestar psicológico: el estrés y la calidad de vida en el contexto laboral. Revista Nacional de Administración, 2, https://revistas.uned.ac.cr/index.php/rna/article/view/285/146.

George, D. y Mallery, P. (2003). SPSS for Windows step by step: A Simple Guide and Reference. 11.0 Update (4. a ed.). Boston: Allyn \& Bacon. http://www.sciepub.com/reference/65129

González-Baltazar R., León-Cortés SG, Contreras-Estrada MI, Hidalgo-Santacruz G., Liliana Hidalgo-González M. (2019) Calidad de vida laboral en trabajadores de la salud en Guadalajara, México. En: Goossens R. (eds) Avances en ergonomía social y ocupacional. AHFE 2018. Advances in Intelligent Systems and Computing, vol 792. Springer, Cham. https://doi.org/10.1007/978-3-319-94000-7_26

Gutiérrez, R. (2017). Recursos Humanos. Recuperado de Como evaluar el desempeño laboral de un empleado: https://factorialhr.es/blog/desempeno-laboral/ 
Hernández-Vicente, I. A., Lumbreras-Guzmán, M., Méndez-Hernández, P., Rojas-Lima, E., Cervantes-Rodríguez, M., \& Juárez-Flores, C. A. (2017). Validación de una escala para medir la calidad de vida laboral en hospitales públicos de Tlaxcala. Salud pública de México, 59, 183-192. Recuperado de

Hernández Sampieri, R, Fernández Collado., \& Baptista Lucio, P. (2014). Metodología de la investigación. Sexta edición. México D.F.

Matabanchoy, S., Alvarez, K., \& Rlobamba, O. (2019). Efectos de la evaluación de desempeño en la calidad de vida laboral del trabajador. Universidad y Salud, 4.

Medina Mendoza, E. J., \& Olivares Saavedra, G. (2018). Modelo de evaluación del desempeño de $360^{\circ}$ para la mejora de la gestión del recurso humano en la Clinica San Lorenzo SRL Cajamarca.

Münch, L. (2015). Fundamentos de la Administración: casos y prácticas de gestión (5a edición). México: Editorial Trillas.

OCDE (2020), ¿Cómo va la vida? 2020: Midiendo el bienestar, Publicaciones de la OCDE. Recuperado de: https://doi.org/10.1787/9870c393-en .

OCDE (2020), ¿Cómo va la vida? 2020: Midiendo el bienestar , Publicaciones de la OCDE, París, https://doi.org/10.1787/9870c393-en .

Organización Internacional del Trabajo (OIT). Trabajo decente para todos. Publicaciones OIT, Recuperado de : https://www.ilo.org/madrid/fow/trabajo-decente-para-todos/lang--es/index.htm

(OIT) Organización Internacional Del Trabajo (2020). https://www.ilo.org/global/about-the-ilo/lang-es/index.htm

Organización Mundial de la Salud (OMS). Principios éticos. Publicaciones OMS. Recuperado de: https://www.who.int/es/about/ethics

Patlán Pérez, J. (2016). Construcción y propiedades psicométricas de la Escala de Calidad de Vida en el Trabajo. Ciencia \& trabajo, 18(56), 94-105. http://dx.doi.org/10.4067/S0718-24492016000200004

Pérez, O. (2015). Formato de Evaluación 360 grados. Blog People next. Recuperado de https://blog.peoplenext.com.mx/formato-de-evaluacion-360-grados

Quintana, M., Carrillo, K., Figueroa, C., Garcia, J., Salazar, R., Tinajero, R., y otros. (2016). calidad de vida laboral de enfermeras de un hospital privado. Biotecnia, 3-4.

Torres, V. (2017). Calidad de vida en el trabajo: antecedentes e importancia de la calidad de vida en el trabajo. Manual moderno.

Zamora Tovar, C. (2011). Implementación de una evaluación de desempeño $360^{\circ}$ en el talento humano gerente de una organización manufacturera.

WHO Quality of Life Assessment Group. (1996). ¿Qué calidad de vida? / Grupo de la OMS sobre la calidad de vida. Foro mundial de la salud $1996 ; 17(4)$ : 385-387 https://apps.who.int/iris/handle/10665/55264

Esta obra está bajo una Licencia Creative Commons

Attribución-NoCommercial 4.0 International

(cc) EY-NC 